\title{
Band Gap Energy in Silicon
}

\author{
Jeremy J. Low, Michael L. Kreider, Drew P. Pulsifer \\ Andrew S. Jones and Tariq H. Gilani \\ Department of Physics \\ Millersville University \\ P. O. Box 1002 \\ Millersville, Pennsylvania 17551 USA
}

Received: December 26, 2007

Accepted: April 16, 2008

\begin{abstract}
The band gap energy $E_{g}$ in silicon was found by exploiting the linear relationship between the temperature and voltage for the constant current in the temperature range of $275 \mathrm{~K}$ to $333 \mathrm{~K}$. Within the precision of our experiment, the results obtained are in good agreement with the known value energy gap in silicon. The temperature dependence of $E_{g}$ for silicon has also been studied.

\section{INTRODUCTION}

The study of the band gap structure of a semiconductor is important since it is directly related to its electronic properties. As a consequence, it has attracted a

where $a=\frac{e}{k C}$ and $b=\frac{-E_{g}}{k C}$. Hence,

$$
E_{g}=-\left(\frac{b}{a}\right) e .
$$
\end{abstract}
considerable interest in undergraduate laboratories [1-3]. Several different methods have been discussed to determine the band gap energy of semiconductors [1-4]. A diode is also used as a temperature sensor [5], in which the linear relationship between the temperature, $T$ and the forward voltage drop across the p-n junction, $V$ is exploited.

It has been shown that with certain simplifications, the $T-V$ relationship for a diode can be written as [4]

$$
T=\left(\frac{e}{k C}\right) V-\left(\frac{E_{g}}{k C}\right)
$$

where $e$ is the charge of electron, $k$ is Boltzmann constant, $E_{g}$ is band gap energy and $C$ is a function of $I$, the current through the diode. Therefore, if $I$ is kept constant, then

$$
T=a V+b
$$

Therefore, if we find constants $a$ and $b$ by experimentally measuring the temperature and voltage across the diode, we can find the band gap energy of a semiconductor, provided that $E_{g}$ does not depend strongly on temperature. For silicon, the variation of $E_{g}$ with temperature is weak [6] in the temperature range of our interest, $273 \mathrm{~K}$ to $335 \mathrm{~K}$.

\section{EXPERIMENTAL METHODS}

Figure 1(a) shows the schematic diagram of the circuit used for $T-V$ data measurements for the base-collector junction of an npn transistor (MPS2222AG) while Figure 1(b) depicts the use of a silicon diode (1N914). The current, I was kept constant using the circuit [7] shown in the dotted box in Figure 1(a). The variable resistor was adjusted to obtain $10 \mu \mathrm{A}$ current. The whole circuit was powered by a constant voltage power supply (Elenco Precision, Model XP-660), keeping the 


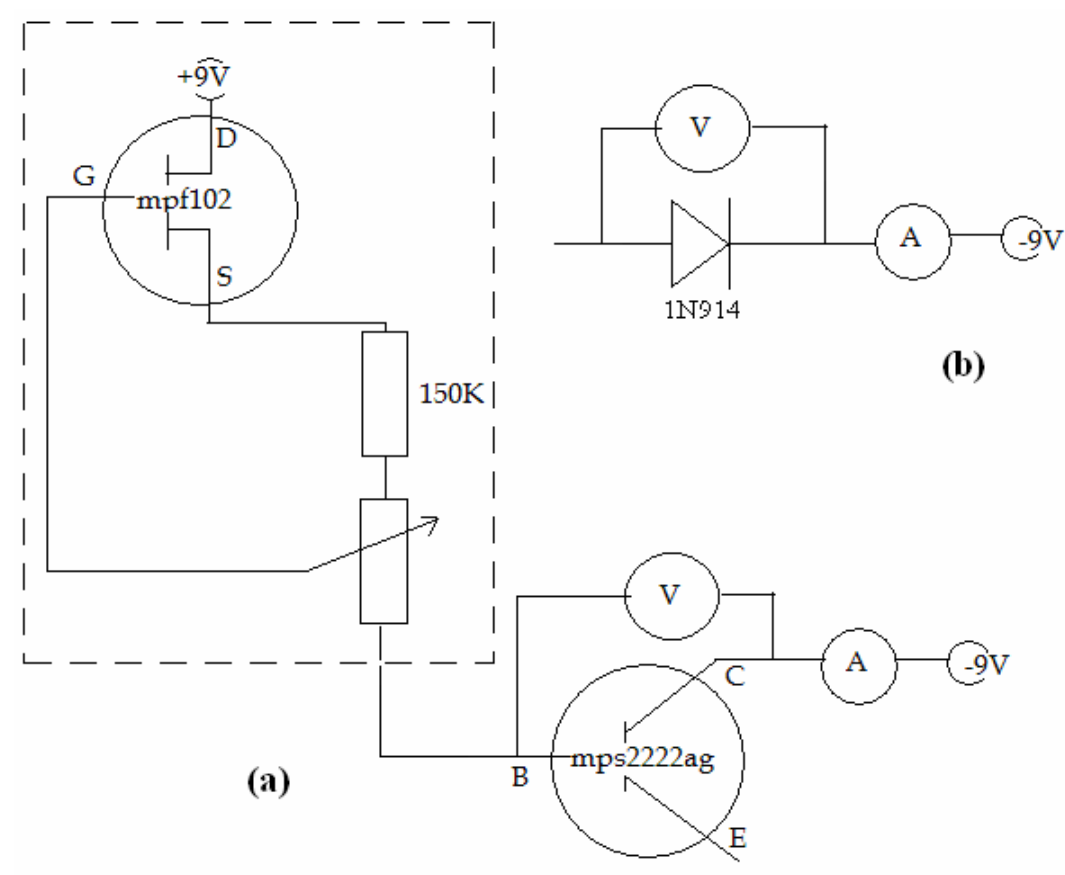

Figure 1. (a) The MPS2222AG Transistor and (b) the 1N914 Diode are at variable temperature. The MPF102 transistor was used as a constant current source. The circuit for constant current source is shown in dotted box above. The gate-source bias voltage was adjusted by a $100-\mathrm{k} \Omega$ variable resistor to obtain a constant current of $10 \mu \mathrm{A}$.

voltage at approximately $9 \mathrm{~V}$. The voltage drop across the pn-junction was measured by a digital voltmeter (Heath, Model SM2320), while the temperature was measured by a carefully calibrated AD- 590 temperature sensor. Both diode and temperature sensors, were wrapped together in a thin plastic foil to insulate them electrically. The whole system was then placed in a de-ionized ice-water mixture (the ice was also made from de-ionized water) to obtain the temperature of $273 \mathrm{~K}$.

The voltage readings were then taken for various temperatures while the system was heated slowly. The measurements were repeated several times to reduce the statistical errors.

\section{RESULTS}

The $T-V$ curve for the silicon pn junction of transistor (MPS2222AG) in the temperature range of $273 \mathrm{~K}$ to $335 \mathrm{~K}$ is shown in Figure 2(a). The data exhibits a fairly good linear relationship between absolute temperature and the voltage drop across the pn junction in the temperature range of our interest, except at the higher end of temperature (see the Discussion). We also used a $1 \mathrm{~N} 914$ silicon diode in place of the transistor (MPS2222AG) and repeated the experiment. The $T-V$ data for the silicon diode (1N914) is shown in Figure 2(b). The values of constants $a$ and $b$ of Eqn. (2) were obtained by the leastsquares fit of the data from $275 \mathrm{~K}$ to $330 \mathrm{~K}$ temperature. The value of $E_{g}$ was then calculated from Eqn. (3).

The uncertainties in results were found in the following way. Since the calculation of $E_{g}$ depends on two variables, $a$ and $b$, the uncertainty in $E_{g}$ is written as $d E_{g}=\left(\frac{\partial E_{g}}{\partial b}\right) d b+\left(\frac{\partial E_{g}}{\partial a}\right) d a$. The maximum fractional error is therefore $\frac{d E_{g}}{E_{g}}=\varepsilon=\left|\frac{d b}{b}\right|+\left|\frac{d a}{a}\right|$, where $d a$ and $d b$ are standard deviations of the slope, $a$ and 

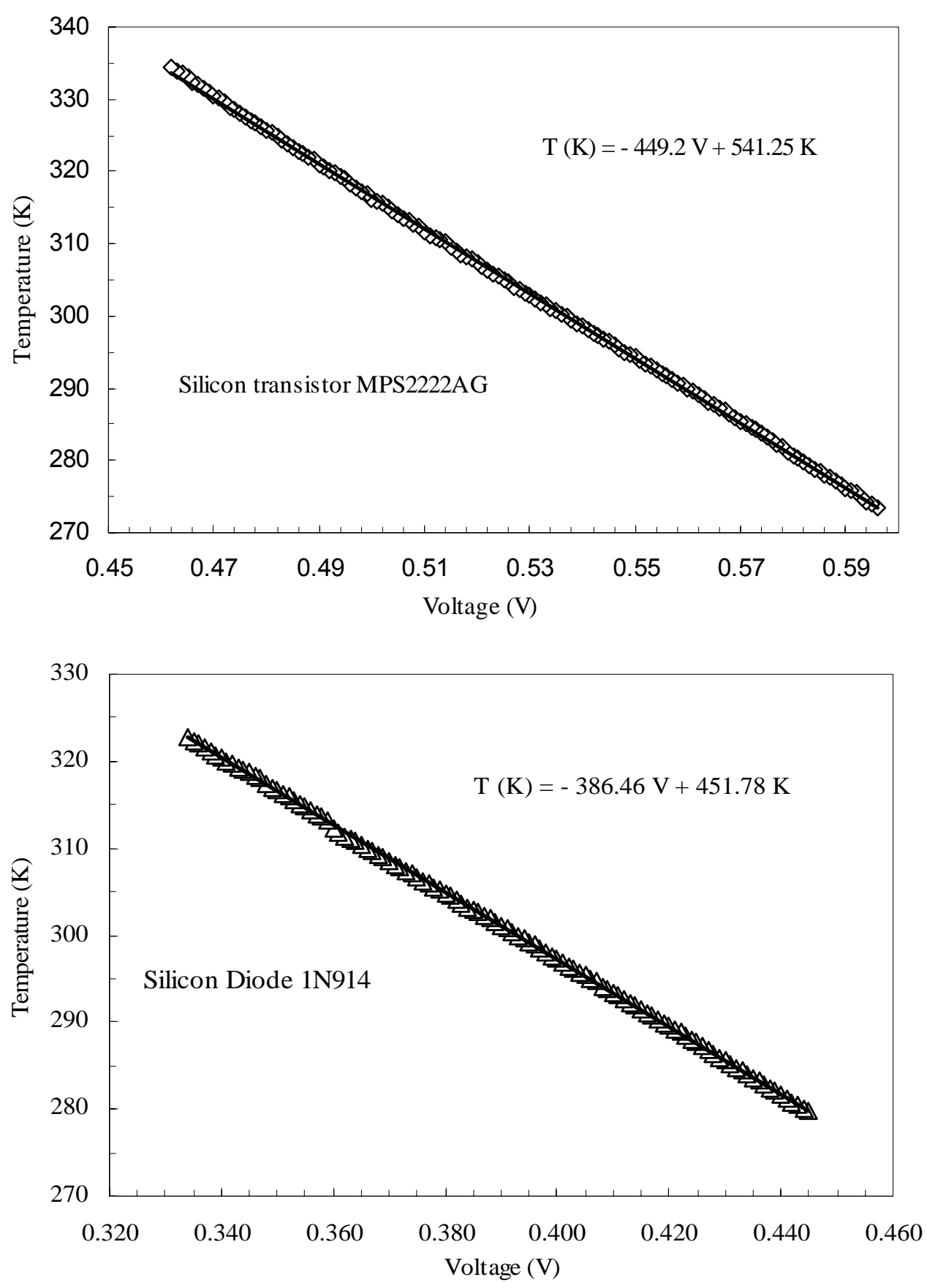

Figure 2. Temperature vs voltage graphs for the silicon: (a, at top) pn junction of npn transistor (MPS2222AG). The T-V curve becomes nonlinear above $330 \mathrm{~K}$. (b, at bottom) diode (1N914). Solid line is the least-squares fit to the data.

y-intercept, $\quad b$ of straight line fit to $T-V$ data, respectively.

For the pn junction of the MPS2222AG transistor [see Figure 2 (a)], $a$ $=-462.17 \mathrm{~K} / \mathrm{V}, d a= \pm 7.95 \mathrm{~K} / \mathrm{V}, \quad b=$
$547.40 \mathrm{~K}$, and $d b= \pm 4.12 \mathrm{~K}$, Therefore in the temperature range of $273 \mathrm{~K}$ to $330 \mathrm{~K}$, the value of $E_{g}$ is $1.20 \mathrm{eV}$ with uncertainty of $\pm 2.47 \%$. Similarly for the 1 N914 diode 


\begin{tabular}{|c|c|c|c|c|c|}
\hline & $a(\mathrm{~K} / \mathrm{V})$ & $b(\mathbf{K})$ & $\begin{array}{c}E_{g}(\mathrm{eV}) \\
273 \mathrm{~K} \text { to } 330 \mathrm{~K}\end{array}$ & $\begin{array}{c}E_{g}(\mathrm{eV}) \\
288 \mathrm{~K} \text { to } 383 \mathrm{~K} \\
\text { [Ref. 4] }\end{array}$ & $\begin{array}{c}E_{g}(\mathrm{eV}) \\
300 \mathrm{~K} \\
\text { [Ref. 6] }\end{array}$ \\
\hline MPS2222AG & $\begin{array}{c}-462.17 \\
\pm 7.95\end{array}$ & $\begin{array}{l}547.40 \\
\pm 4.12\end{array}$ & $\begin{array}{c}1.20 \\
\pm 0.03\end{array}$ & \multirow{2}{*}{1.23} & \multirow{2}{*}{1.12} \\
\hline 1N915 & $\begin{array}{c}-393.54 \\
\pm 6.38\end{array}$ & $\begin{array}{l}454.18 \\
\pm 2.39\end{array}$ & $\begin{array}{c}1.17 \\
\pm 0.03\end{array}$ & & \\
\hline
\end{tabular}

Table 1. Linear regression coefficients $a$ and $b$ obtained from measured $T-V$ data, and $E_{g}$ calculated from them. Last two columns are $E_{g}$ values at given temperature from Ref. 4 and Ref. 6 , for comparison.

[see Figure 2(b)], $a=-393.538 \mathrm{~K} / \mathrm{V}, \quad d a=$ $\pm 6.3828 \mathrm{~K} / \mathrm{V}, b=454.18 \mathrm{~K}$, and $d b= \pm$ $2.388 \mathrm{~K}$. Hence in the temperature range of $278 \mathrm{~K}$ to $323 \mathrm{~K}$, the value of $E_{g}$ is $1.17 \mathrm{eV}$ with uncertainty of about $\pm 2.15 \%$. The results are summarized in Table 1 and compared with known values of energy gap in silicon from different references.

For silicon the value of $E_{g}$ is known within $\pm 5 \%$ uncertainty and varies from 1.13 $\pm 0.057 \mathrm{eV}$ at $273 \mathrm{~K}$ to $1.11 \pm 0.056 \mathrm{eV}$ at $330 \mathrm{~K}[6,8]$. Therefore our results are in good agreement to the known values in literature $[4,6,8]$, although the values obtained in here tend to be slightly higher (see Discussion).

We have further analyzed our data to investigate the temperature dependence of $E_{g}$. The straight line was obtained by the least-squares fit of the $T-V$ data in small temperature intervals of $\pm 2.5 \mathrm{~K}$, assuming that $E_{g}$ of silicon remains constant in small temperature intervals. The results are shown in Figure 3.

\section{DISCUSSION}

\section{a. Temperature - Voltage Curve}

As shown in Figure 2 (a) $T-V$ curve deviates from a straight line towards higher temperature side $(>330 \mathrm{~K})$. Such non-linear behavior in $T-V$ curve was also observed in Germanium above $313 \mathrm{~K}$ [4]. When deriving equation (2) using the Ideal Diode Equation [4, 6]:

$$
I=I_{0}\left[e^{(e V / k T)}-1\right]
$$

where $I_{0}$ is reverse saturation current, we assumed $e^{(e V / k T)}>>1$ to obtain

$$
I=I_{0} e^{(e V / k T)}
$$

For a forward bias voltage of $0.4 \mathrm{~V}$, taken at room temperature, we get

$$
e V / k T=0.4 / 0.025=16 \text {, }
$$

thus

$$
e^{e v / k T} \approx 8.9 E+6
$$

Therefore, the approximation is generally justified at room temperature and below. But as temperature increases the exponential term decreases. This may cause a deviation from the linear behavior of $T-V$ data.

The reverse current $I_{0}$ depends strongly on temperature. It is proportional [6] to the Boltzmann factor $\exp \left(-E_{g} / k T\right)$ and to $T^{3+\gamma / 2}$, where $\gamma$ is a constant. Therefore, 


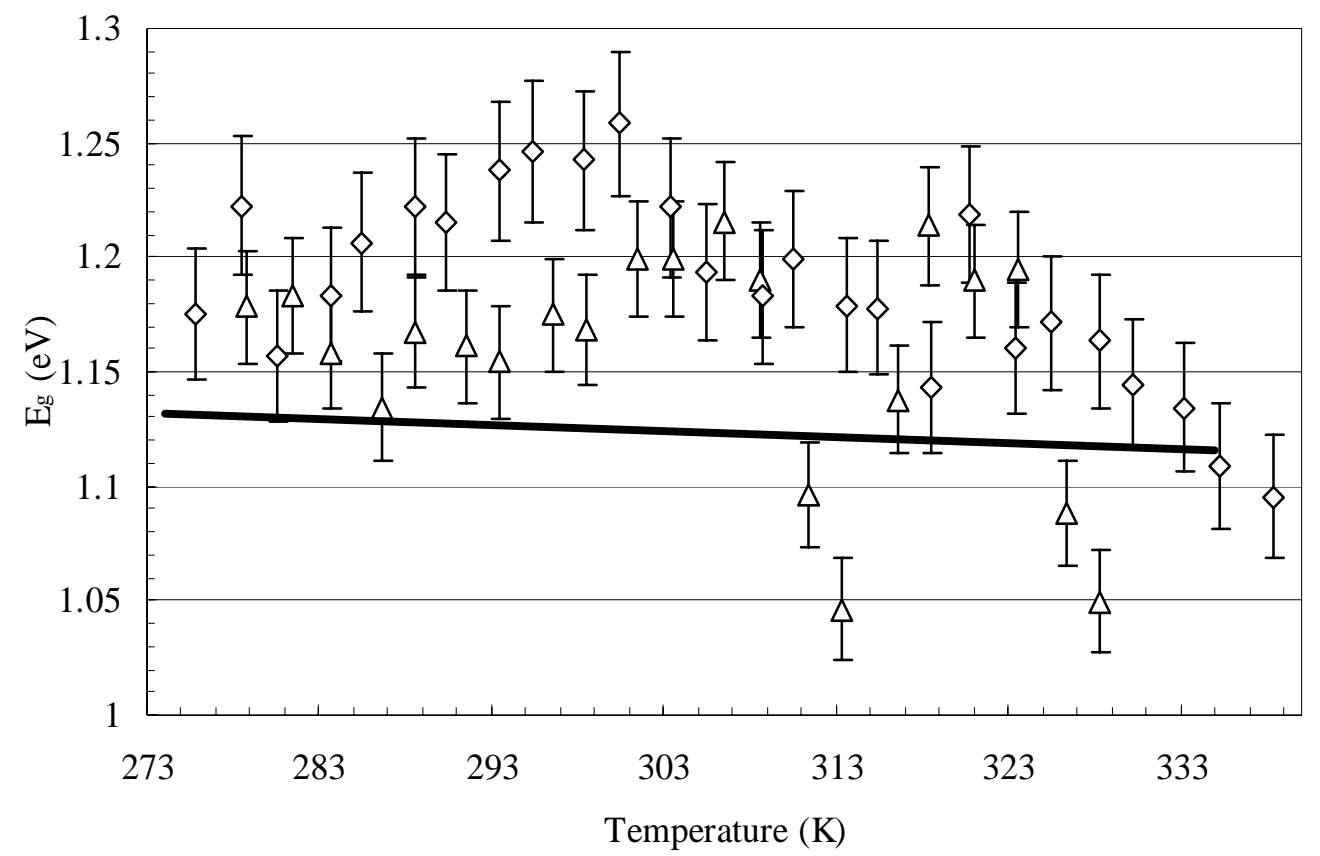

Figure 3. The temperature dependence of the band gap energy in silicon: $\diamond$ the pn junction of MPS2222AG npn transistor, $\Delta$ 1N914 diode, and solid line represents the universal function taken from Ref. 6 for comparison.

$$
I_{0}=A T^{3+\gamma / 2} \exp \left(-E_{g} / k T\right)
$$

Here $T^{3+\gamma / 2}$ dependence of $I_{0}$ is neglected as compared to its exponential dependence on $T$ and thus

$$
I_{0}=B \exp \left(-E_{g} / k T\right)
$$

where $B=A T^{3+\gamma / 2}$. Now combining Eqn. (5) and Eqn. (7), we get

$$
I=B \exp \left(-E_{g} / k T+e V / k T\right)
$$

Since $I$ is maintained constant in the experiment, therefore we can write Eqn. (1) in which $C=\ln (I / B)$.

b. Values of $E_{g}$

But if $T^{3+\gamma / 2}$ dependence is not ignored, then the so-called constant $C$ is no more a constant and will depend on $\ln (T)$. That means the experimental slope of
$T-V$ graph will be smaller and hence the value of $E_{g}$ obtained here is expected to be slightly higher. Similar results are reported for silicon as well as for germanium in Ref. 4 (see table 1). Also the contribution of $\ln (T)$ term will be larger towards higher temperature and this may also contribute in non-linear behavior of $T-V$ data at higher temperature.

\section{c. Temperature Dependence of $E_{g}$}

So for we have assumed that $E_{g}$ of silicon is temperature independent which is not exactly true. As a matter of fact $E_{g}$ does depend on the temperature, although weakly as described by a universal function [6] and is shown in Fig. 3 (solid line). To investigate the temperature dependence of $E_{g}$, the straight line was obtained by the least square fit of the data in the temperature intervals of $\pm 2.5 \mathrm{~K}$, assuming 
that $E_{g}$ of silicon remains constant in small temperature intervals.

In the derivation of Eqn. (2), the $T^{3+\gamma / 2}$ term was neglected as compared to the exponential dependence of temperature. For a temperature change $\pm 2.5 \mathrm{~K}(\Delta T=$ $5 \mathrm{~K})$ at room temperature, the contribution of exponential term is almost ten times higher than that of the $T^{3+\gamma / 2}$ term (Assuming $\gamma=$ 1 for simplification). Therefore this assumption can also be justified in small temperature intervals.

As shown in Fig. 3, the temperature dependence of $E_{g}$ in silicon is weak and is consistent with the description of the universal function [6]. The value of $E_{g}$ in silicon obtained in our experiments varies from about $1.18 \mathrm{eV}$ at $275 \mathrm{~K}$ to $1.1 \mathrm{eV}$ at $335 \mathrm{~K}$. The data for npn-transistor (MPS2222AG) exhibits a small peak in $E_{g}$ between the temperature range of about 290 $\mathrm{K}$ to $300 \mathrm{~K}$ (see Fig. 3). We intend to offer no explanation for this behavior at this stage. Note that the data is more scattered towards higher temperature range as expected and is explained earlier. The temperature dependence of $E_{g}$ given by universal function [6] is plotted in solid line varies in Fig. 3 for comparison.

\section{CONCLUSION}

The band gap energy, $E_{g}$ of silicon temperature range of $273 \mathrm{~K}$ to $335 \mathrm{~K}$ was investigated. At $275 \mathrm{~K}$ temperature it is found to be $1.18 \pm 0.03 \mathrm{eV}$ and it weekly depends on the temperature. The data is in good agreement with the earlier known values in the literature $[4,6,8]$.

\section{ACKNOWLEDGEMENT}

The authors are thankful to Dr. John Dooley for valuable discussions and suggestions. We also wish to acknowledge the technical help of Mr. Shawn Reinfried at different stages of the experiment.

\section{REFERENCES}

1. A. Sconza, G. Torzo, and G. Viola, Am. J. Phys. 62, 66-70 (1994).

2. P. J. Collings, Am. J. Phys. 48, 197-199 (1980).

3. B. D. Sukheeja, Am. J. Phys. 51, 72 (1983).

4. J. W. Precker, and M. A. da Silva, Am. J. Phys. 70, 1150-1153 (2002).

5. P. R. N. Childs, J. R. Greenwood, and C. A. Long, Rev Sci. Inst. 71, 295-2978 (2000).

6. S. M. Sze, The Physics of Semiconductor Devices, (Wiley, New York, 1969) pp. 12-20.

7. Thomas L. Floyd, Electronics Devices, $7^{\text {th }}$ Ed., (Prentice Hall, New Jersey, 2005) pp.718.

8. N. W. Ashcroft, and N. D. Mermin, Solid State Physics, (Holt, Rinehart and Winston, Philadelphia, 1976) pp. 566. and its temperature dependence in the

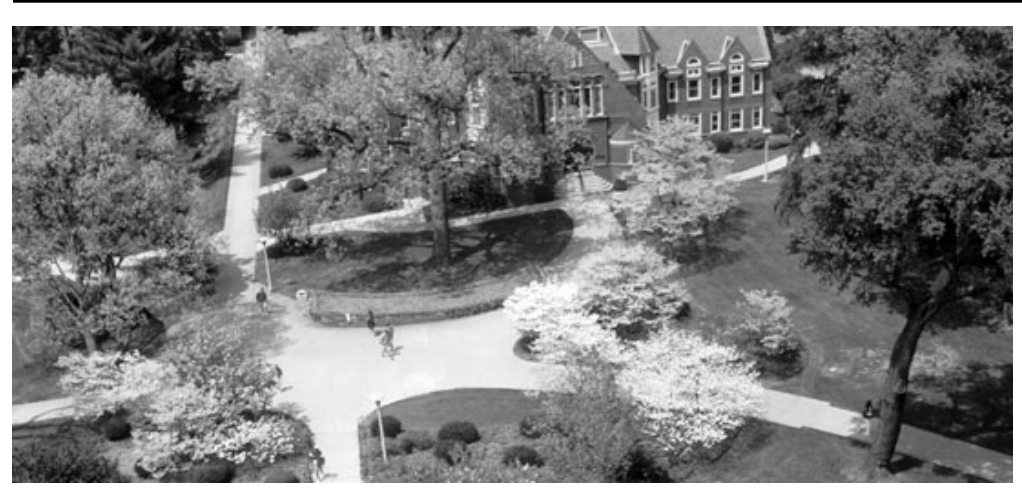

Millersville University is the best choice for people of Pennsylvania and beyond who are motivated to apply their native intelligence and a world-class education to the challenging and important work of guiding themselves, their families and their communities through times of changing opportunity. The men and women of the Millersville University faculty are scholars highly respected in their fields; approachable teachers and active mentors who engage their students in the classroom, the office, the research lab, and in the life of the campus. The faculty is thoroughly supported in its work by the men and women of the Millersville University staff and leadership team-caring people of competence and integrity who share with their faculty partners a determination that Millersville graduates shall be as prepared to lead robust intellectual, professional and civic lives as the graduates of any of the nation's best known institutions.

www.millersville.edu 